\title{
Leading Change in a 150-year-old Medical School: Overcoming the Challenges of a VUCA World Amidst a 21 st Century Pandemic
}

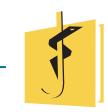

\author{
Ma. Lourdes P. Domingo-Maglinao, MD
}

This oration was delivered by the author during the annual discurso de apertura at the formal opening of the academic year 2021-2022 at the University of Santo Tomas last August 2, 2021 . The discurso started as a Dominican Catholic tradition on July 16, 1866 as an annual event.[1] The professor or dean assigned the preparation of this academic paper is appointed by the rector of the university a few months prior to the opening of each school year.[2]

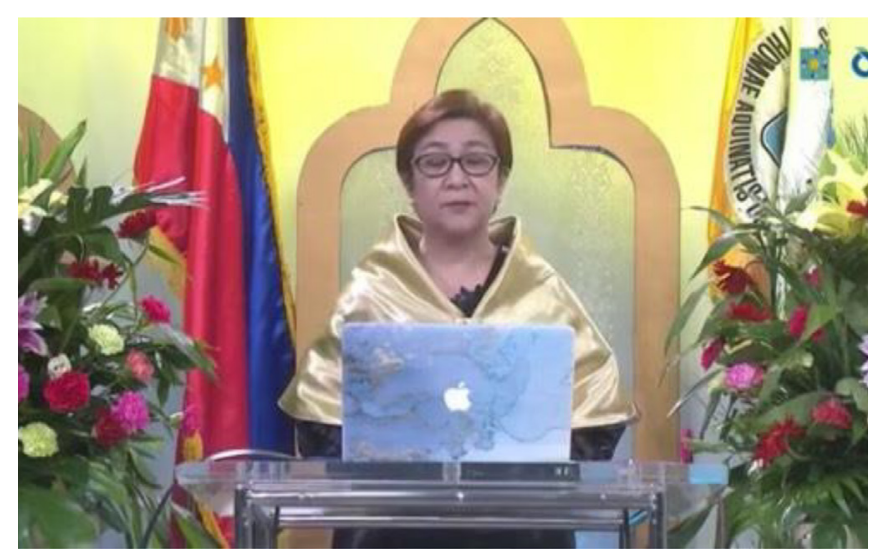

Ma. Lourdes P. Domingo-Maglinao, MD mdmaglinao@ust.edu.ph

Dean, Faculty of Medicine and Surgery, University of Santo Tomas, Espana, Manila, Philippines
"And like the moon, we must go through phases of emptiness to feel full again."

Anonymous

I believe in dreams. As far as my memory serves me, as a young girl, I dreamt of being in space as an astronaut, fascinated with the moon, the suit, huge aircrafts, and anti-gravity experiences. Once, though very short-lived, I dreamt of being a soldier in a war zone. I loved watching "Combat," starring Vic Morrow and Rick Jason (some of you may relate). However, everything changed when I was 10 years old when I got fixated on becoming a doctor, a neurosurgeon... my profession... my vocation... my true love.

I believe in life paths. The Lord led me to where I am today. Call it serendipity or destiny, but to this day, I still regard myself as a fortuitous, shy, and reluctant administrator. I have envisioned myself being an astronaut in space, a soldier on the battlefield, a surgeon in the operating room, but never in my wildest dreams did I imagine myself where I am positioned today, taking this enormous amount of courage, leading a 150-year-old medical school in a VUCA world in the midst of a 21 st century pandemic... and this is what I call my lifepath. I am equally blessed to have been given this opportunity to speak as the $153^{\text {rd }}$ Orator at the Discurso de Apertura today during the opening of AY 2021-2022. I follow the path 
of other illustrious speakers before me in this UST Catholic tradition that started on July 16, 1866.

\section{THE VUCA WORLD}

Today, nearly 2,000 Thomasian medical students are dreaming of their own destinies and life paths, and to them, the UST Faculty of Medicine and Surgery (FMS) owes much as they will be our link to the future. As the story of the generations is shaped by the formative events that unfold during their times, the story of our medical students today is shaped by the ongoing COVID-19 pandemic.

As we draw our breaths in these globally unprecedented and immensely disruptive times, we are reminded of how VUCA (Volatility, Uncertainty, Complexity, and Ambiguityl in the future will inescapably persist in being. Yet, interestingly, VUCA is a military coinage, perhaps not the military of my youth wherein I imagined myself as a mere soldier, but one that I can now relate with as an administrator, the General, so to speak, of a 150-year-old medical institution of higher education.

We are immersed in a VUCA world wherein volatility demands that we react with agility, uncertainty requires purpose-driven action, complexity urges us to adopt a dynamic mindset, and ambiguity challenges us to redirect our vision and mission. Eventually, I realized that the learnings wrought by the VUCA world undeniably push us to embrace more agile, flexible, and adaptive governance models so that we may deal with exponential transformation in medical education and beyond. The COVID-19 pandemic created fractures and fissures in the medical education system. Everyone is caught in the midst of a perfect battlefield as it unmasked the hidden fragilities of the system and vulnerabilities of all its stakeholders. The abrupt and urgent need to change triggered a shift of seismic proportion over a very short period of time.

As in all healing professions, Medicine is among the hardest hit. Our faculty staff are front liners battling their own personal stresses, fearful that they may be infected or transmit the infection to family and loved ones. We deal with patients suffering from COVID infections on a daily basis. An added stress is seeing colleagues succumbed to the dreaded disease.

\section{VUCA and the Transformative Change in Medical School During the Pandemic}

John Kotter, perhaps the most respected thinker when it comes to change leadership mentioned the need to create an urgency for change as the first step in his 8-step change model. Yet, the VUCA scenario bypassed this step and defined the speed of change that became the driving force of the FMS. We needed to recognize the uncertainties and inescapable force early on so that we may move relentlessly. We were plunged into a climate that left us gasping. Therefore, it was imperative to navigate assiduously, institute adaptive measures in the delivery of medical education during these transformative times, and become more future-equipped.

The elements of VUCA are not new and have been around for quite some time. Still, the COVID-19 pandemic clearly escalated, intensified, and protracted these elements, thus posing a clear and present danger to medical education. Therefore, its relevance has never been felt as much as it is today, and because of the environment that we are immersed in, there is a need to institute instantaneous change. One of the challenges that it presented was prioritizing our learners' and staff's safety, health, and wellness while ensuring integrity and continuity of the medical education process and generating uncompromised learning outcomes. The challenge of producing future doctors despite the current situation remains a daunting task. Several other overwhelming challenges we experienced mainly affected the delivery of instructions. Obstacles were related to delivery in the traditional format, the need to rapidly adapt to virtual platforms, and overcome economic repercussions. A surge in the number of cases affected the deployment of our clinical clerks for core clinical training. Data gathering for conducting research was likewise strained.

\section{The Medical School as an Ecosystem Within the University Ecosystem}

Sound pedagogical principles anchored on advanced educational technology are at the core of every higher education institution. The surge in COVID-19 cases forced an abrupt shift that affected workplace fluidity. All processes must instantly switch into digital spaces.

As part of the university ecosystem, the FMS benefitted from the vision of past and present 


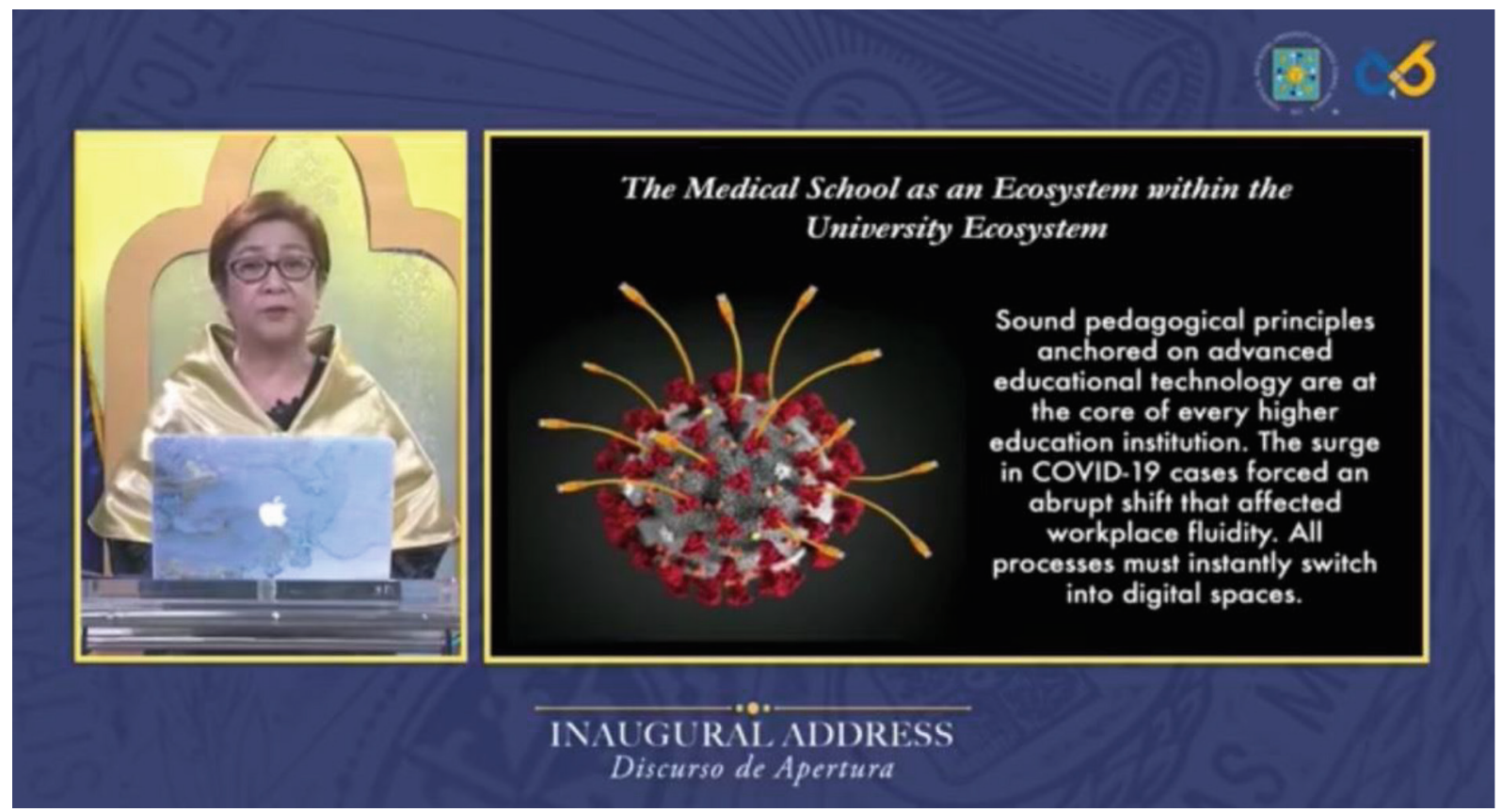

university officials who had the foresight to invest in educational media and technology, the Blackboard LMS, and the human resources to support it back in 2003. The Rapid elearning Training Programs instituted by the university since 2012 retooled our education workforce even before the pandemic, thus allowing the medical school and all faculties and colleges to successfully and seamlessly navigate into the fully online mode of instructions posed by the pandemic. Despite the unexpected impositions of lockdowns, we were able to anchor technological solutions to ensure continuity of education. The Office of the Vice-Rector for Academic Affairs, the Center for Innovative Teaching and Educational Delivery, or the (CITED) and the pedagogical leads, the UST Technology Center and the Exemplary League of Innovative Thomasian Educators (ELITE) that comprised the e-learning specialists of the university deployed to the various faculties and colleges mustered pedagogy-driven and technologydriven trainings through the Enriched Virtual Mode of Instruction (EMV) and the Teaching and Innovation Goals for E-learning Redesign or the (TIGER) Training at the UST Cloud Campus that became the cornerstone, the rock upon which the weight of the entire educational structure at UST rests.

\section{Embracing the New Normal}

As we braved the challenges of coexisting with the pandemic, we embraced the new normal, focused on identifying and addressing the most important issues at hand. It made us zero in on innovation, resilience, and perseverance more than what we have done at any other time. It provided us with the environment to reimagine - engage on what works and abandon what doesn't. But there was no path to follow and that was the struggle, a trial-and-error experience, a hit and miss scorecard leading to what can be the footprint of medical education in the new normal.

Our clinical clerkship is by far the most affected. What is supposed to be a 12-month hospital rotation turned into a virtual clerkship experience. As a team, the FMS employed a project-planpractice approach and ventured into risks by adopting the double loop approach to learning. Double-loop learning as a concept and process developed by Chris Argyris and Donald Schon (1978) is different from single-loop learning, which involves changing methods and improving efficiency to obtain established objectives (ie, "doing things right"). Through double-loop learning, the team challenged their long-held beliefs and assumptions and changed objectives themselves (ie, "doing the right things"). In the first loop - it meant to harness critical thinking and decision-making processes through case history-taking, virtual PE to arrive at the best possible diagnosis; and in the second loop, which was expected to supplement and strengthen the learnings from the first loop, flexibility in learning was introduced through virtual and actual skills laboratory, and simulation, while adhering to 
and remaining fully compliant with minimum requirements set by the Commission on Higher Education. Several innovative strategies to address the challenges were instituted; allow me to share some of them with you.

\section{Virtual patient encounters}

During history-taking and physical examination facilitated by technology, students formulated the correct clinical diagnoses and created appropriate management plans. The virtual encounters facilitated proper decorum and communication skills with actual patients.

\section{Telemedicine as the new normal}

Future doctors learned web side manners to triage patients based on specific needs. Bringing the clinic right into the patient's own home to mitigate disease transmission.

\section{Telemedicine in the Community}

We continued to break barriers to access of care via telemedicine services with 33 partner communities in the midst of the pandemic. Virtual home visits to chronically ill individuals through assistance by community workers. Exercise of social accountability carried out through advocacy capstone projects and enhancement of primary care interventions and communication skills via Objective Structured Clinical Examination on health education counseling, roleplaying on breasffeeding counseling, and introduction of public health interventions. During the pandemic, we have completed 115 community advocacy projects - on health, inclusive education and literacy, spiritual enrichment, and environmental health.

\section{Virtual Clinical Rounds}

The pandemic threatened to topple down another tradition in the field of medicine, the medical rounds. Medical rounds comprise the signature pedagogy in medicine, a form or style of teaching and instruction that is common to the specialties of medical profession. Often cutting across individual courses and institutions, signature pedagogies help students build "habits of mind" that allow them to think and act in the same manner as the experts in the field, insofar as it seeks to hone students' inductive and deductive analytical skills.

Hence, the simulated medical rounds were conceptualized using the constructivist approach, which asserts that people construct their own understanding and knowledge of the world through experiencing things and reflecting on those experiences. Medical clerks in cardiology rotation experienced daily rounds using simulated patients wherein they experienced writing daily Subjective, Objective, Assessment, and Plan or the SOAP notes based on progressive disclosures of the patients' signs and symptoms, including analysis of laboratory results, interpretations of ECGs, and giving rationale pharmacological and non-pharmacological regimens. The faculty assigned for the day gave the debriefing. The medical clerks then wrote their reflections about the experience in the virtual cardiology ward.

\section{Research}

Research generation has remained the fundamental metric underpinning the performance of all medical schools. Our senior medical students generated an average of 100 scientific research articles last year alone. The designs of the different researches have been adapted to the exigencies of time: scoping reviews, systematic reviews and meta-analysis; translation and validation studies of research instruments; and qualitative studies that were amenable to online focus group discussions in clinical epidemiology and preventive medicine; and educational studies based on ongoing educational interventions, as well as, various clinical topics in specialties and subspecialties of medicine and surgery are just some of the adaptations that were undertaken at the height of the pandemic. The annual clinical clerks research paper presentation pushed through via the virtual platform.

\section{Virtual Skills Assessment}

Virtual evaluation of clinical skills was done through Direct Observation of Procedural Skills (DOPS) and Objective Structured Clinical Examination (OSCE) including communication and professional conduct while role playing with household members as "patients". Students' ingenuities are tapped as they are strongly encouraged to innovate. 
The ePortfolio

The ePortfolio is a learning and assessment tool of $21^{\text {st }}$ century skills. It allows the student to: organize, save, document, publish, and digitally share his work as an accurate assessment tool. Developmental in nature, it shows the levels of individual progress in learning over time periods (documents of the learning journey of students that may include a personal reflection that cannot be documented in traditional assessment methods). It is an objective evaluation tool that will enable the faculty to carry out a continuous, objective, and accurate assessment of authentic skills, and can be relied upon to evaluate performance according to quality standards. The ePorffolio is concerned with assessing all aspects of the learner with several tools included in the file and provides an opportunity for improved performance and adaptability. Moreover, it contributes to the development of personal skills and experiences of both the faculty and learner.

\section{Online Oral Revalida}

The COVID-19 pandemic threatened to topple down a 144-year-old valuable academic tradition of the FMS. Not even war, nor any crisis has ever prevented the discharge and execution of this exercise since 1877. As academic leaders, we must harness the collective wisdom of all the faculty and come up with a strategic alternative to this culminating academic exercise.

The logistical challenge of the Revalida has been present since the in-person setting. Converting it to virtual needs re-imagination of how this can be delivered in a seamless and valid manner considering the resources and proficiency of both faculty and students. Staging the fully Online Oral Revalida was like an orchestra, that required close seamless collaboration of several offices and departments in the midst of uncertain times while we only project, plan, and prepare virtually. The first challenge is getting everyone on the same page with both old and new faculty, novice, and advanced users of technology. Varying degrees of technology adoption, resistance, and infrastructure problems make the deployment a logistical nightmare. To date, we have successfully launched two Online Revalidas. Motivating, educating, and training every participant in this noble exercise is a feat of its own.
In 2020 and now 2021, there were 447 and 483 graduating medical candidates respectively, who underwent the Online Revalida with 240 faculty members that comprised 148 and 161, 3-member tribunals. There were 150 and 180 clinical cases prepared by eight departments namely: Internal Medicine, Surgery, Obstetrics and Gynecology, Pediatrics, Otorhinolaryngology, Ophthalmology, Dermatology, and Family Medicine, and these cases were submitted to and meticulously reviewed by the Department of Medical Education. The Blackboard Learning Management System coupled with the Google productivity suite both under the UST Cloud Campus System allowed for a virtual convergence of the faculty and students. The COVID-19 pandemic created an auspicious opportunity to innovate a century-old academic assessment tradition utilizing a critical and flexible approach, timely and adaptive strategy, and the value of collaborative effort and creativity is of great importance.

\section{Resumption of Face-to-Face Clerkship}

Behind the successful transition and being one of the first medical schools granted authority to conduct clinical clerkship thus paving the way for other medical and allied health programs to follow suit, were grueling "seems - like - forever" behind the scenes months of thoughtful, tedious, meticulous planning, preparations, and dry runs to allow a safe re-engagement of our clinical clerks using innovative strategies for teaching and learning (TL). It may seem so simple, especially to those who only see the seamless finished product of this one colossal task. Reliving the journey makes me palpitate but the decision to take the calibrated risk and proceed with the giant leap is something we shall never regret. Creation of clinical rotations, clerkship guidelines, health, and safety protocol, retrofitting of facilities, endless documentary requirements, application, and evaluation procedures and securing approvals from Local Government Unit (LGU), Department of Health Inter-Agency Task Force (DOH-IATF), Commission on Higher Education (CHED) to painstakingly sourcing vaccines for the clinical clerks as early as possible and preparation of contingency plans were all a product of team collaboration among the FMS, Office of the Vice-Rector for Academic Affairs, Office of the Vice-Rector for Finance, Office of the Secretary-General, UST Health Service, Santo 
Tomas e-Service Providers (STEPs), Office of Public Affairs, Security Office, UST Hospital, and Facilities and Management Office. The FMS cannot thank you all enough for the full support you have given us. With this, the FMS is now the benchmark of other medical schools that intend to resume limited faceto-face clerkship.

\section{Continuing Medical Education}

Our office for Continuing Medical Education continues to host numerous local and international webinars, postgraduate courses, and special memorial lectures all in celebration of our $150^{\text {th }}$ year even at the height of the pandemic. The USTMed 150 Webinar Series is a series of 150 regular online lectures or panel discussions featuring esteemed alumni here and abroad to talk about topics of their expertise in their respective field or specialty, as well as topics regarding their passion and life outside of medicine. This USTMed150 webinar series will serve as a prelude to the sesquicentennial celebration's culminating activity, the "Summit of Thomasian Global Health Leaders".

\section{Strengthening Inner Qualities as Change Leaders}

As physician leaders, we tread two spheres. As physicians committed to compassionate patient care, we spend most years of our lives practicing and refining our clinical specialty. Competent leadership is a particular discipline that exceedingly varies from clinical competencies of which physicians devote so much time to acquire. There are specific skills and adeptness developed and harnessed in our traditional medical training that leaves out opportunities for learning leadership competencies.

Mindfulness is such an important component of leadership competency. As a neurosurgeon, I know how our brain works... persistent 'mindfulness,' to be ready to shift and adapt to the set of specific operating principles of the clinical and organizational milieu. Mindfulness leads to the strengthening of inner qualities as a change leader. Thus, building individual resilience.

Then comes the disruptive change and being plunged into the current pandemic situation, thus confronted with incessant paradoxes and strains that make decision-making a labyrinth of what-ifs. Thrown in a circumstance where we must strike a balance between deliberate immediate actions and short- to medium-term judicious endeavors, we realized that long-term future planning cannot be achieved, given the current predicament. As we engaged in so much innovation to strive for normalcy, we learned that one innovative strategy would transform and lead to another; thus, moving us farther away from the conventional. And as we provide optimal solutions to the disruptions brought by the pandemic to medical education, we find ourselves in a scuffle to resolve between optimizing merits while streamlining costs. Perhaps the pivotal insights I gained from this crisis is a people-centered leadership that focused on the soft skills of leadership, ie, adaptive innovation, team coalition, and interdependence.

\section{Fostering the Right Conditions to Create Inclusivity}

As a subsystem of the university, the FMS has the technology and infrastructure, and human resources provided by educational media and technology experts to support the creation of online course sites in our blackboard learning management systems. However, we also acknowledged that we needed to support the faculty, learners, and families during these pandemic times. Not all of them have the same access to information and communication technologies (ICTs), which vary greatly across different areas of the country.

Therefore, we supported the model proposed by Daniel Stanford, author of the "Bandwidth Immediacy Matrix," which suggests that instructors use low-bandwidth tools that nevertheless lend themselves to a sense of immediacy like collaborative documents and group chat. Zoom eventually became synonymous with synchronous learning a virtual classroom that goes beyond geographical boundaries and enables learning beyond the traditional model. Zoom has afforded the university and the FMS flexibility to continue teaching and enable learners to engage in online education. For the most vulnerable students with unstable connections who might not have access to synchronized digital learning activities, the recordings of the lecture and pdf files of PowerPoint presentations are uploaded in the course sites. Asynchronous activities are given to complement the "live" sessions. Other modes of communication using SMS, FB Messenger, and 
Viber, are also utilized for immediate response and feedback.

\section{Building Individual Resilience}

In these challenging times posed by unprecedented global public health emergency, medical educators are responsible for medical undergraduates, postgraduate interns, trainees, and clinicians throughout the professional lifecycle, ie, undergraduate, graduate education, and continuing professional development.

The medical students and faculty staff faced significant health risks and concomitant stress, fear, decreased sense of control, and uncertainty. Early on, we were cognizant that a deleterious impact on both physical and mental health could result, especially for our faculty staff, who had the added burden to work shifts in the hospitals to care for COVID-19 patients. Changes in governmental policies and hospital protocols for healthcare professionals and disruption of educational formats and requirements for trainees took place.

At all stages of their professional lifecycle, our medical faculty staff had to deal with the difficult anxiety-provoking realities of this public health crisis. Members of our faculty staff are frontline healthcare professionals involved in caring for many seriously ill patients. Moral distress, including difficult care decisions, personal health risk, and/ or potential risk of transmitting the infection to one's family, posed a significant burden to their mental health. Moreover, they are faced with the realities of colleagues who succumbed to the infection. Educational disruptions created significant anxiety for medical clerks about completing requirements and achieving competencies before graduation. Significant financial burden confronted several of our students and staff that we literally solicited from generous sources in order to bridge the financial gaps.

Within this, the crisis created an imperative for medical faculty staff to strengthen their own coping skills while supporting students' well-being through adaptive flexibility for curriculum innovation and culturally sensitive resilience and well-being interventions. Strategies to optimize resilience and wellbeing with an integrative resilience approach of individual, learning environment, organization, and system factors were instituted. Townhall and numerous pocket meetings were called to address problems and provide immediate solutions.

\section{Harnessing Collective Energy}

Sensemaking is a critical leadership skill wherein educators structure their own understanding of an ambiguous situation. As a cognitive process, it aims at educators' understanding of unfamiliar or unexpected situations that may arise in an institution. With the reinvention of the educational platform caused by the COVID-19 pandemic, sensemaking becomes vital for educators to comprehend the situation to frame significant actions in response to the dilemmas this new educational ecosystem has posed.

Involvement of the FMS in this immense effort to innovate and eventually change is NOT the sole responsibility of its leader. We have identified and acknowledged each and everyone's strength and accountability, thus becoming an all-member engagement. This is the true essence of transformative change which is critical and highly participatory in the process. The FMS needed to shift mainly to a culture of teamwork, creativity, and innovation entailing a high level of commitment and skills collaboration. This is all about appreciating interdependence. This does not solely involve cerebral participation but engages the heart, our deep aspirations as well, activating not only the power of collective purpose but inviting to greater compassion.

Despite the seemingly insurmountable challenges, we are optimistic that we shall emerge as victors in this race. FMS's successful transition is never a singular achievement of its leadership but rather a collective one. I believe in modeling selfless leadership as well as collective leadership as it is the path to sustainability. Transformative leadership is collective. Ultimately, the culture of organizational resilience and agility that we have gained becomes the bedrock of all our successful endeavors.

\section{An Eye into the Future}

The UST FMS offers an innovative preparatory path to the degree of Doctor of Medicine, the LearningEnhanced Accelerated Program for Medicine, or simply, LEAPMed, is our response to the growing number of preparatory years for medicine brought about by the $K$ to 12 program. This is the 
contribution of university to the increasing demand for medical human resources in the country. Offered only to academically exceptional students who are committed to proceeding to medicine proper, we have graduated our first class of 76 , all of them with Latin honors.

The Organ Donation Innovative Strategies for Southeast Asia (ODISSeA) project is a specialized postgraduate certificate program in organ donation. We are one of only eight southeast Asian beneficiary universities, pioneers in offering a training program for transplant specialists designed by the University of Barcelona, the only academic institution offering a master's program in organ donation in Europe. This certificate program is highly endorsed by the Department of Health among its hospitals, as well as in accredited private hospitals involved in transplantation, as it is directed towards its goal of improving transplantation rates and outcomes in the country, consistent with the Philippine health agenda.

Our Center for Pain Medicine and Thomasian Audiologists continued to be the prime movers in pain medicine and assisting Government Hearing Health and Prevention of Deafness Program through pioneering research, technical assistance, and capacity building activities. We have a 100\% employment rate, $40 \%$ locally and $60 \%$ overseas.

The UST FMS did not miss the opportunity to capitalize on our 150 years' history of quality medical education to partner with The Nuffield Centre for International Health and Development at the University of Leeds for our Master in Public Health International Program through the British Council and Commission on Higher Education collaborative project, Joint Development of Niche Programmes through Philippine-UK Linkages. Recently, our MPH I is one of only seven chosen Phil HEls to be given scholarship grants in 2022 by the Access and Competitiveness through International Higher Education or the ACT-IHE in partnership with the Commission on Higher Education.

The Journal of Medicine, UST (JMUST) is the reinvented scholarly publication of the UST FMS as it brings "light" to the generation of promising young Thomasian researchers who again have been blessed with the "voice" to be heard and a "venue" to be read. This tool for timely dissemination and assimilation of research-based discoveries shall continue the tradition of excellence in medical education and research in partnership with the
USTH. JMUST is in the one-last step to SCOPUS but we are already at Repositories and Registries now of: CrossRef-CitedBy (w/ our DOl-Digital Object Identifier-for individual Author ownership), Western Pacific Regional Index Medicus (WPRIM), HERDIN Plus, ResearchGate, Google Scholar, and Semantic Scholar.

The FMS, being the first in Asia to integrate ultrasound in the medical curriculum, whose vision is for ultrasound, is regarded as the stethoscope of the future, to be used to its full potential and for every medical student to have this handheld tool in their pockets. We shall soon launch our USTFMS PoCUS (Point of Care Ultrasound) Training Institute, which will equip the 21 st-century physicians in the country with relevant skills to respond to the evolving global health care needs and enable them to deliver service of greater worth. This is an enterprise that merges transformative education and the merits of this valuable tool that effectively impacts patient service at the point of care. We also envision this to be an avenue for interprofessional education and collaboration among health allied programs.

Very recently, through the initiative of the FMS, the university has forged an auspicious alliance with the local government by making the university an official vaccination site for the city of Manila. We envisioned this to be the impetus of a strong and lasting partnership, and a model of a governmentprivate engagement in the trust of community service and social accountability. This will open new doors for further collaboration, especially in medicine and health allied disciplines. As part of social responsibility, our faculty and clinical clerks are now serving as volunteer vaccinators and clinical screeners in UST and other vaccination sites, inculcating the virtue of service to others before self, most especially during these challenging times. This is the university and the FMS's contribution to humanity as we do our share in fighting this pandemic.

Our heartfelt gratitude and credit go to our benevolent university officials for believing in us and sharing the dream. One of our two landmark projects, the redevelopment of the rear portion of St. Martin de Porres building is nearing completion. This will definitely complement the teaching and learning environment of the 21 st century medical education. We owe the university, tremendous funding of our digital marketing campaign which is the first among medical schools in the country and is currently being 
deployed. No Philippine medical school is privileged to have a 150-year foundation anniversary and taking off from this opportunity, this pioneering project uses the FMS sesquicentennial celebration as the pivot of this social media intervention. It is envisioned that this bold endeavor will continue to build, nurture, and engage all our stakeholders, especially our alumni and other partners as we continue to be the trailblazer not only in the country but also in the region and perhaps in the world. Gratitude turns what we have into enough, and for every grateful heart, proof that we can turn a vision into fruition and bring a dream into reality.

Simulation as a tool for teaching and learning is a cornerstone of any outcomes-based mindset. Today, top-performing medical schools worldwide have long embraced simulation to amplify real patient experiences in the first few years of healthcare education. The Sts. Cosmas and Damian Simulation and Research facility aims to strengthen the interface between medical education and actual clinical practice of our medical learners and assure ethical and compassionate patient care and the safety of both the patient and healthcare provider. This second landmark project is a milestone and a dream come true that will usher and herald us to a future that will perpetuate the Thomasian brand of excellence in medicine over the next 150 years and beyond.

Allow me to give credit to every person who supported us and appreciate every Thomasian alumnus who shared and believed in this dream, being steadfast in their commitment, sharing their lifelong blessings, unfaltering in their support and magnanimity for the advancement and furtherance of Thomasian medical education to keep us at par with the world's best.

As we chart the direction of our medical school in the years following our 150th foundation, we are driven by the principles and philosophy of the great Thomasian imprint, which espouses competence, commitment, and compassion in the pursuit of a legacy that we can live and later leave. There is nothing predetermined about the history of human existence because new trajectories are made when we challenge the status quo. This is the essence of the sesquicentennial spirit that we all took to heart.

Allow me to read an excerpt from my Commencement Ceremonies Message to our UST Medicine Class of 2021 - "... Every person's life is blessed with victories and strengthened by defeats. What makes life truly worth living may not necessarily reside in the days we found glory and triumph, but rather in the struggles we overcame to sustain and survive. The years of formative medical schooling have been defined by your own set of personal struggles and challenges. This pandemic did not make things easier. In fact, it did the opposite. Living in it and though it has been one constant challenge that every man must confront and accept. It is not easy, but it can be done. It is not simple, but it is possible. Remember that nobody is without troubles and genuine challenges. The fact is, that it is not obvious because we seldom advertise our innermost woes and heartaches. But do know, that no one

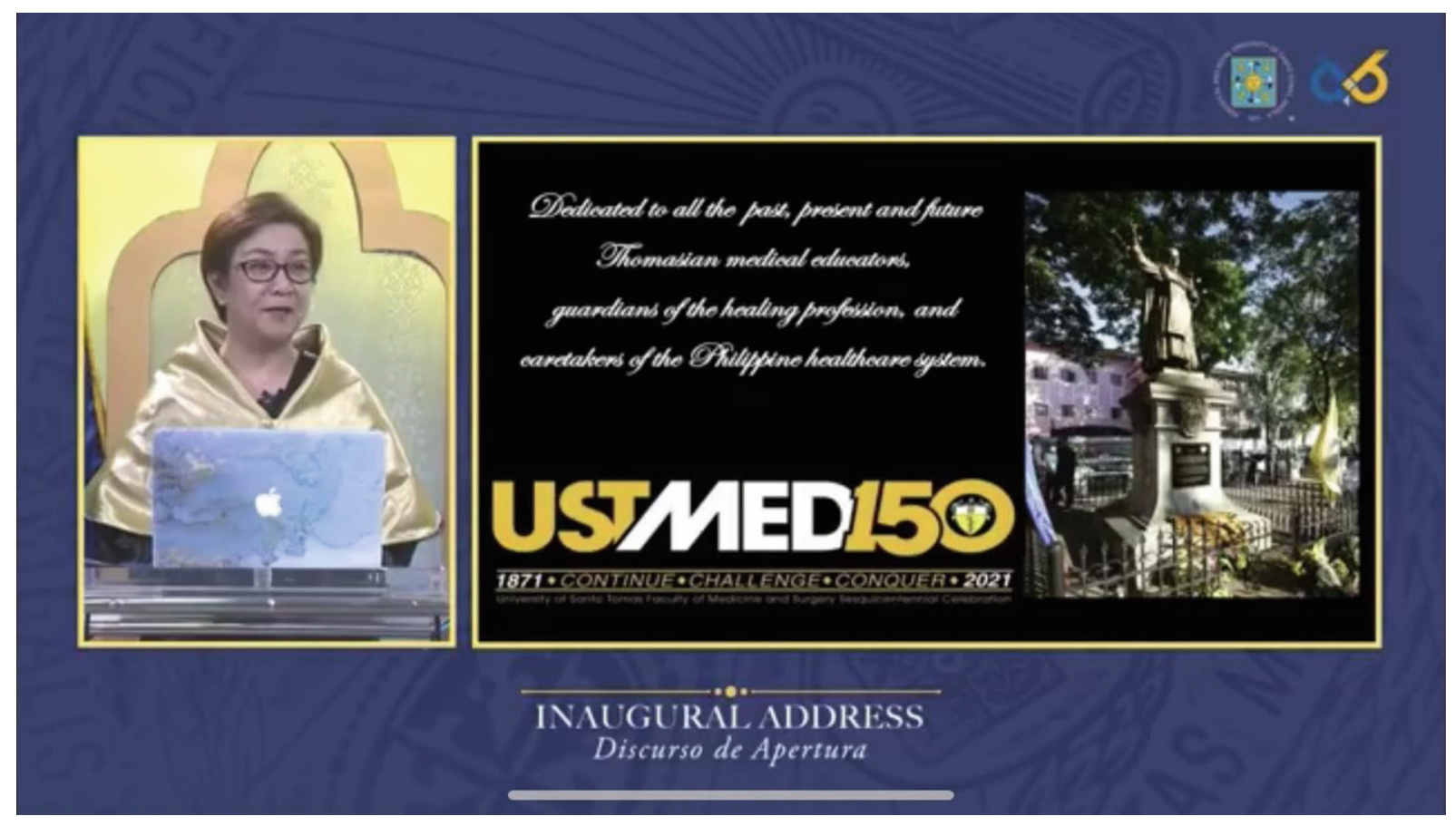


escapes challenges, not even the purest of hearts with the purest intentions, survived life without the scars of fiercely fought battles. But you should not fear challenges. They must be experienced to find meaning in what we value in life. They must be lived through, so we can validate the impermanence of its impact. They must be surmounted to prove that challenges only become obstacles when we bow to it. Face the challenges that will come your way and do not make them rule your days. Look at them as opportunities to be better, brighter, and bolder and see their dark side and difficult lessons of obstacles, as vessels that shine the greatest gems of light ..."

The ground is shifting beneath us and change is ongoing. But even so, our foundation at the UST FMS is solid, built upon the age-old ideals of our illustrious predecessors of some 150 years. I dare say, we are fortunate that we are living through this historic change! Today we are not just living history; instead, we are shaping it... fostering innovation in Philippine medical education, so that future Filipino Thomasian physicians will be better prepared for the realities of healthcare in the 21 st century. The FMS remains unstoppable and will continue to be the epitome of excellence in medical education.

The COVID pandemic threatened to topple us in many ways, but with our indomitable spirit, we prevailed."

Let me end with this quote from Colin Kaepernick, an American civil rights activist, and former football quarterback... "Don't Ask If Your Dreams Are Crazy; Ask If They're Crazy Enough."

Dedicated to all the past, present, and future Thomasian medical educators, guardians of the healing profession, and caretakers of the Philippine healthcare system.

Thank you. 


\section{REFERENCES}

1. Pe JL. The University of Santo Tomas in the twentieth century. University of Santo Tomas Press; 1973.

2. De Ramos NV. I walked with twelve UST rectors. Quezon City: A.G. Ablaza and C. de Ramos Ablaza; 2000.

(c) (1) (2) Open Access This article is licensed under a BY NC SA Creative Commons Attribution-NonCommercialShareAlike 4.0 International License, which permits use, share - copy and redistribute the material in any medium or format, adapt - remix, transform, and build upon the material, as long as you give appropriate credit, provide a link to the license, and indicate if changes were made. You may do so in any reasonable manner, but not in any way that suggests the licensor endorses you or your use. You may not use the material for commercial purposes. If you remix, transform, or build upon the material, you must distribute your contributions under the same license as the original. You may not apply legal terms or technological measures that legally restrict others from doing anything the license permits. The images or other third party material in this article are included in the article's Creative Commons license, unless indicated otherwise in a credit line to the material. If material is not included in the article's Creative Commons license and your intended use is not permitted by statutory regulation or exceeds the permitted use, you will need to obtain permission directly from the copyright holder. To view a copy of this license, visit https://creativecommons.org/licenses/by-nc-sa/4.0/. 